\title{
Associação entre baixos níveis de aptidão física e fatores sociodemográficos em adolescentes de área urbanas e rurais
}

\author{
Association between low levels of physical fitness and sociodemographic \\ factors in adolescents from rural and urban areas
}

\author{
E.L. Petroski, A.F. Silva, A.B. Rodrigues, A. Pelegrini
}

ARTIGO ORIGINAL | ORIGINAL ARTICLE

O estudo teve como objetivo verificar a associação entre a aptidão física e fatores sociodemográficos em adolescentes domiciliados em áreas urbanas e rurais. Estudo transversal conduzido em 627 adolescentes (299 urbana e 328 rural), de 14 a 17 anos, de escolas públicas estaduais. Informações sociodemográficas, antropométricas e de aptidão física relacionada à saúde (Physical Best) foram coletadas. Os resultados indicaram que, na flexibilidade, os adolescentes da área urbana apresentaram $56 \%$ mais chance de inadequação em relação aos da área rural; na força abdominal, aqueles domiciliados na área rural apresentaram quase 10 vezes mais chance de inadequação quando comparados aos da área urbana; na aptidão cardiorrespiratória, os adolescentes da área urbana apresentaram $91 \%$ mais chance de inadequação que os da área rural. Concluiu-se que os adolescentes de escolas públicas domiciliados em áreas urbanas são mais expostos a inadequação da aptidão física relacionada à saúde que adolescentes da área rural.

Palavras-chave: adolescente, aptidão física, escolares, saúde

ABSTRACT

The objective was to evaluate the association between low levels of physical fitness and sociodemographic factors in adolescents from rural and urban areas. A cross-sectional study was conducted on 627 adolescents (299 from urban areas and 328 from rural areas) aged 14 to 17 years, who attended state public schools. Sociodemographic data, anthropometric measures and healthrelated physical fitness data (Physical Best) were collected. With respect to flexibility, adolescents from urban areas presented a $56 \%$ higher chance of inadequate flexibility than schoolchildren from rural areas. In the sit-up test, adolescents from rural areas presented an almost 10 times higher chance of inadequate muscle strength/endurance than those from urban areas. Regarding cardiorespiratory fitness, adolescents from urban areas showed a $91 \%$ higher chance of inadequate fitness than those from rural areas. In conclusion, adolescents from urban areas attending state public schools are more prone to inadequate health-related physical fitness than adolescents from rural areas.

Keywords: adolescent, physical fitness, schoolchildren, health

Submetido: 10.12.2010 | Aceite: 10.01.2011

Edio Luiz Petroski. Centro de Desportos. Programa de Pós-Graduação em Educação Física; Núcleo de Pesquisa em Cineantropometria e Desempenho Humano; Universidade Federal de Santa Catarina, Florianópolis, Santa Catarina, Brasil.

Adelson Fernandes da Silva e Adriana Bispo Rodrigues. Universidade Estadual de Montes Claros, MG, Brasil.

Andreia Pelegrini. Departamento de Educação Física, Centro de Ciências da Saúde e do Esporte da Universidade do Estado de Santa Catarina, Brasil.

Endereço para correspondência: Edio Luiz Petroski, Centro de Desportos, Universidade Federal de Santa Catarina, Campus Universitário - Trindade - Caixa Postal 476, CEP: 88040-900 Florianópolis, SC, Brasil.

E-mail: petroski@cds.ufsc.br 
Embora exista um número cada vez maior de evidências que comprovam os benefícios da aptidão física para a saúde (Canadian Society for Exercise Physiology, 2004), observa-se que uma grande parcela da população de escolares não apresenta níveis de aptidão física adequada à saúde (Lopes, Maia, Silva, Seabra, \& Morais, 2004; Serassuelo Junior et al., 2005; Silva, Paccini, \& Glaner, 2007).

A crescente urbanização da sociedade brasileira tem sido apontada como a principal causa da baixa aptidão física em escolares, sendo responsável pela diminuição de espaços para a prática de atividade física e esportes. Além disso, o desenvolvimento tecnológico tem propiciado facilidades e comodidades que favorece a adoção de um estilo de vida pouco ativo fisicamente por crianças e adolescentes.

$\mathrm{O}$ que tem chamado a atenção, sobretudo, dos pesquisadores e profissionais das áreas de Saúde e Ciências do Esporte, é a magnitude dos baixos padrões de aptidão física que tem atingido um contingente cada vez maior de crianças e adolescentes, quer seja dos meios urbano ou rural (Glaner, 2002). Seria esperado que escolares residentes em áreas rurais possuíssem estilos de vida mais vigorosos, por estarem expostos a ambientes mais propícios a prática de atividade física.

Há evidências de que a aptidão física relacionada à saúde (AFRS) de crianças e adolescentes seja relacionada ou influenciada pelo meio ambiente em que estes estão inseridos (Canadian Society for Exercise Physiology, 2004). No entanto, um estudo conduzido por Ramos et al. (2008), mostrou que não há predomínio de um único meio sociogeográfico nos componentes da aptidão física. Estudos que analisaram a aptidão física de escolares de alto (Ronque et al., 2007) e baixo nível socioeconômico (Serassuelo Junior et al., 2005) revelaram elevados padrões de AFRS inadequados em áreas urbanas.

Segundo Glaner (2005), pessoas de uma mesma região geográfica, separados por apenas alguns quilômetros, podem ter estilos de vida completamente diferentes no que tange à prática de atividades físicas, principalmente, entre as áreas rurais e urbanas. Fundamentando-se nessas observações, o objetivo do presente estudo foi verificar a associação entre a aptidão física e fatores sócio-demográficos em adolescentes domiciliados em áreas urbanas e rurais.

\section{MÉTODO}

Este estudo, caracterizado como transversal, foi realizado com escolares do meio urbano e rural do ensino público estadual do município de Januária, Minas Gerais, Brasil.

\section{Amostra}

A população do estudo foi composta por 4495 adolescentes matriculados em escolas públicas estaduais. Para o cálculo do tamanho amostral, adotou-se prevalência desconhecida para o desfecho (igual a 50\%), erro tolerável de cinco pontos percentuais, nível de confiança de $95 \%$, efeito de delineamento de 1.5 , acrescentando $15 \%$ para possíveis perdas e recusas. Assim, estimou-se que seria necessário coletar informações de 611 adolescentes. Em virtude das características do processo amostral por conglomerado, participaram da amostra 627 estudantes, sendo 299 da zona urbana e 328 da zona rural, de ambos os sexos, com idade de 14 a 17 anos.

O processo de amostragem utilizou como unidade amostral as escolas do município de Januária e a seleção da amostra foi realizada em dois estágios: no primeiro, selecionaram-se três escolas da zona urbana e cinco escolas da zona rural; no segundo, foram selecionadas, aleatoriamente, seis e quatro turmas das zonas urbana e rural, respetivamente, de cada uma das escolas.

Todos os responsáveis pelos escolares, após serem informados sobre o propósito da investigação e os procedimentos a serem adotados, assinaram um termo de consentimento livre e esclarecido. Este estudo foi aprovado pelo Comitê de Ética em Pesquisa da FUNORTE Faculdades Unidas do Norte de Minas (Parecer 129/09). 


\section{Instrumentos e Procedimentos}

Foram coletadas informações sociodemográficas (sexo, idade, nível econômico e área de domicílio), antropométricas (massa corporal e estatura) e AFRS (flexibilidade, força/resistência muscular e aptidão cardiorrespiratória).

O nível econômico (NE) foi verificado por meio do critério de classificação da Associação Brasileira de Estudos Populacionais (2008). Os escolares foram classificados em três categorias: alto $(\mathrm{A} 1+\mathrm{A} 2+\mathrm{B} 1+\mathrm{B} 2)$, médio $(\mathrm{C})$ e baixo $(\mathrm{D}+\mathrm{E})$.

A aferição antropométrica seguiu o protocolo da Canadian Society for Exercise Physiology (2004). A massa corporal (MC) foi obtida em balança digital, da marca Filizola, com resolução de $0.05 \mathrm{~kg}$, e a estatura (EST) por meio de uma trena antropométrica da marca Sanny, com resolução de $0.1 \mathrm{~cm}$, fixada na parede. A partir dessas informações, obtevese o índice de massa corporal [IMC $=\mathrm{MC}(\mathrm{kg}) /$ $\left.\operatorname{EST}^{2}\left(\mathrm{~m}^{2}\right)\right]$. Para o estado nutricional foram utilizados os pontos de corte para adolescentes, considerando idade e sexo (Cole, Bellizzi, Flegal, \& Dietz, 2000; Cole, Flegal, Nicholls, \& Jackson, 2007). Para classificar o estado nutricional foram utilizados os valores de IMC equivalentes a adultos, como segue: baixo peso $\left(\right.$ IMC $\left.<18.5 \mathrm{~kg} / \mathrm{m}^{2}\right)$, eutrófico (IMC $=18.5 \mathrm{a}$ $<25.0 \mathrm{~kg} / \mathrm{m}^{2}$ ) e excesso de peso (IMC $>25$ $\left.\mathrm{kg} / \mathrm{m}^{2}\right)$.

A AFRS foi obtida por meio da aplicação de uma bateria de testes de motores, a qual foi aplicada obedecendo à seguinte ordem: "sentare-alcançar" (flexibilidade), flexão abdominal modificado 1 minuto (força/resistência muscular) e corrida/caminhada de $1600 \mathrm{~m}$ para o indicador a aptidão cardiorrespiratória (American Alliance for Health, Physical Education, Recreation and Dance - AAHPERD, 1988). A AFRS foi classificada em adequada e inadequada de acordo com os critérios de referências recomendados pelo Physical Best (AAHPERD, 1988).

\section{Análise Estatística}

Os dados foram tratados por meio de esta- tística descritiva (distribuição de frequências) e inferencial (qui-quadrado e regressão logística). O teste qui-quadrado foi utilizado para verificar possíveis associações entre as variáveis. A regressão logística foi utilizada para verificar associação entre os desfechos inadequados (flexibilidade, força/resistência muscular e aptidão cardiorrespiratória) e as variáveis independentes (sexo, faixa etária, NE, área de domicílio e estado nutricional). $\mathrm{Na}$ análise bruta, foram calculadas as razões de odds (RO) e o intervalo de confiança de $95 \%$ (IC 95\%) para cada variável de exposição, em relação a uma categoria de referência. Na sequência, o modelo foi ajustado para todas as variáveis independentes. Em todas as análises utilizouse um nível de significância de $5 \%(p<.05)$.

\section{RESULTADOS}

A Tabela 1 apresenta a proporção de escolares com inadequação em cada componente da AFRS de acordo com as variáveis sociodemográficas (sexo, faixa etária, $\mathrm{NE}$ e área de domicílio) e estado nutricional (IMC). Foi encontrada associação entre a flexibilidade, a aptidão cardiorrespiratória e a força/resistência muscular somente com a área de domicílio ( $p$ $<$.05). Na flexibilidade e aptidão cardiorrespiratória, maior proporção de inadequação foi encontrada nos escolares da área urbana, enquanto na força/resistência muscular, proporção mais elevada foi verificada nos da área rural.

A associação entre a flexibilidade inadequada e os fatores sociodemográficos é apresentada na Tabela 2. Foi encontrada associação entre o desfecho e a área de domicílio, na qual os escolares da área urbana apresentaram $55 \%$ (IC 95\% $=1.12-2.12)$ mais chance de inadequação nesse teste motor quando comparado aos da área rural. Quando a análise foi ajustada para todas as variáveis, notou-se que a área de domicílio permaneceu associada à flexibilidade inadequada. Esses resultados revelaram que os escolares da área urbana apresentaram 56\% (IC $95 \%=1.12-2.16)$ mais chance de inadequação da flexibilidade em relação aos adoles- 
8 | E.L. Petroski, A.F. Silva, A.B. Rodrigues, A. Pelegrini

Tabela 1

Proporção (\%) de inadequação dos componentes da aptidão física em escolares por sexo, faixa etária, NE, área de domicílio e estado nutricional

\begin{tabular}{|c|c|c|c|c|}
\hline & & Flexibilidade & $\begin{array}{c}\text { Força/resistência } \\
\text { muscular }\end{array}$ & $\begin{array}{c}\text { Aptidão } \\
\text { cardiorrespiratória }\end{array}$ \\
\hline \multicolumn{5}{|l|}{ Sexo } \\
\hline Masculino & & 39.1 & 97.7 & 35.3 \\
\hline Feminino & & 42.4 & 99.2 & 35.5 \\
\hline & $p$ & .409 & .138 & .975 \\
\hline \multicolumn{5}{|l|}{ Faixa etária } \\
\hline $14-15$ & & 37.8 & 98.8 & 35.1 \\
\hline $16-17$ & & 44.5 & 98.3 & 35.8 \\
\hline & $p$ & .090 & .634 & .850 \\
\hline \multicolumn{5}{|l|}{ Nível econômico } \\
\hline$A+B$ & & 37.7 & 98.7 & 41.6 \\
\hline $\mathrm{C}$ & & 39.9 & 98.2 & 31.8 \\
\hline $\mathrm{D}+\mathrm{E}$ & & 44.0 & 98.8 & 34.8 \\
\hline & $p$ & .417 & .852 & .147 \\
\hline \multicolumn{5}{|l|}{ Área de domicílio } \\
\hline Urbana & & $46.5^{*}$ & $97.3^{*}$ & $43.1^{*}$ \\
\hline Rural & & 36.0 & 99.7 & 28.4 \\
\hline & $p$ & .008 & .013 & $<.001$ \\
\hline \multicolumn{5}{|l|}{ Estado nutricional } \\
\hline Peso normal & & 39.2 & 98.8 & 35.5 \\
\hline Baixo peso & & 46.2 & 97.8 & 38.5 \\
\hline Excesso de peso & & 49.0 & 98.0 & 28.6 \\
\hline & $p$ & .231 & .725 & .503 \\
\hline
\end{tabular}

Nota: ${ }^{*} p<.05$

Tabela 2

Associação da flexibilidade inadequada com fatores associados

\begin{tabular}{|c|c|c|c|c|}
\hline & RO (IC 95\%) & $p$ & RO* (IC 95\%) & $p$ \\
\hline \multicolumn{5}{|l|}{ Sexo } \\
\hline Masculino & 1.0 & & 1.0 & \\
\hline Feminino & $1.15(.83-1.58)$ & .409 & $1.14(.80-1.64)$ & .468 \\
\hline \multicolumn{5}{|l|}{ Faixa etária } \\
\hline $14-15$ & 1.0 & & 1.0 & \\
\hline $16-17$ & $1.39(.96-1.81)$ & .090 & $1.49(.74-3.00)$ & .259 \\
\hline \multicolumn{5}{|l|}{ Nível econômico } \\
\hline$A+B$ & 1.0 & & 1.0 & \\
\hline $\mathrm{C}$ & $1.01(.85-2.08)$ & .660 & $.98(.62-1.56)$ & .945 \\
\hline $\mathrm{D}+\mathrm{E}$ & $1.30(.83-2.68)$ & .210 & $.88(.38-2.08)$ & .778 \\
\hline \multicolumn{5}{|l|}{ Área de domicílio } \\
\hline Urbana & $1.55(1.12-2.12)$ & .008 & $1.56(1.12-2.16)$ & .008 \\
\hline Rural & 1.0 & & 1.0 & \\
\hline \multicolumn{5}{|l|}{ Estado nutricional } \\
\hline Peso normal & 1.0 & & 1.0 & \\
\hline Baixo peso & $1.32(.85-2.08)$ & .217 & $1.32(.84-2.09)$ & .225 \\
\hline Excesso de peso & $1.49(.83-2.68)$ & .189 & $1.51(.84-2.74)$ & .172 \\
\hline
\end{tabular}

Nota: RO - Razão de odds; RO* - RO ajustada para todas as variáveis da tabela 
centes escolares da área rural.

A Tabela 3 apresenta os resultados da análise de regressão logística do teste força/ resistência muscular inadequado em relação aos fatores sociodemográficos. Houve associação apenas entre o desfecho e a área de domicílio. Esses resultados demonstraram que as chances dos adolescentes apresentarem inadequação nessa variável foi 8.99 (IC 95\% = 1.12-72.30) vezes maior naqueles da área rural quando comparado aos da área urbana. Quando a análise foi ajustada, percebeu-se que a área de domicílio permaneceu associada ao desfecho, na qual os escolares da área rural apresentaram quase 10 vezes (IC 95\% $=1.20-$ 83.20) mais chance de inadequação da força/ resistência muscular em relação àqueles da área urbana.

A Tabela 4 apresenta a associação da aptidão cardiorrespiratória inadequada com os fatores sociodemográficos. Assim como nas outras variáveis, a aptidão cardiorrespiratória esteve associada somente à área de domicílio. Esses achados apontaram que os adolescentes residentes na área urbana apresentaram 91\% mais chance de inadequação nessa variável que aqueles da área rural.

\section{DISCUSSÃO}

Este estudo teve como objetivo principal verificar a associação entre aptidão física e fatores sociodemográficos em adolescentes das áreas urbana e rural, tornando-se o primeiro a ser realizado no estado de Minas Gerais, Brasil.

Os componentes da AFRS estiveram associados à área de domicílio, revelando que maior inadequação de flexibilidade e aptidão cardiorrespiratória foi constatada nos adolescentes da área urbana; enquanto maior inadequação da força/resistência abdominal foi verificada naqueles da área rural. Em estudo realizado com adolescentes rurais e urbanos do Norte gaúcho e Oeste catarinense, a maior inadequação dos componentes da AFRS força/resistência muscular e aptidão cardiorrespiratória foi encontrada nos adolescentes da área urbana, enquanto a flexibilidade foi similar entre as áreas de domicílio (Glaner, 2005).

Tabela 3

Associação da força/resistência abdominal inadequada com fatores associados

\begin{tabular}{|c|c|c|c|c|}
\hline & RO (IC 95\%) & $p$ & RO* (IC 95\%) & $p$ \\
\hline \multicolumn{5}{|l|}{ Sexo } \\
\hline Masculino & 1.0 & & 1.0 & \\
\hline Feminino & $2.75(.68-11.11)$ & .155 & $3.21(.64-16.26)$ & .158 \\
\hline \multicolumn{5}{|l|}{ Faixa etária } \\
\hline $14-15$ & $1.38(.37-5.18)$ & .635 & $1.34(.15-12.35)$ & .797 \\
\hline $16-17$ & 1.0 & & 1.0 & \\
\hline \multicolumn{5}{|l|}{ Nível econômico } \\
\hline$A+B$ & 1.0 & & 1.0 & \\
\hline $\mathrm{C}$ & $.72(.13-3.98)$ & .700 & $.73(.09-5.76)$ & .764 \\
\hline $\mathrm{D}+\mathrm{E}$ & $1.08(.18-6.56)$ & .930 & $.79(.04-17.40)$ & .880 \\
\hline \multicolumn{5}{|l|}{ Área de domicílio } \\
\hline Urbana & 1.0 & & 1.0 & \\
\hline Rural & $8.99(1.12-72.30)$ & .039 & $9.99(1.20-83.20)$ & .033 \\
\hline \multicolumn{5}{|l|}{ Estado nutricional } \\
\hline Peso normal & 1.0 & & 1.0 & \\
\hline Baixo peso & $.55(.11-2.79)$ & .475 & $.55(.10-2.88)$ & .477 \\
\hline Excesso de peso & $.60(.07-5.07)$ & .640 & $.68(.08-5.99)$ & .727 \\
\hline
\end{tabular}

Nota: RO - Razão de odds; RO* - RO ajustada para todas as variáveis da tabela 
Tabela 4

Associação da aptidão cardiorrespiratória inadequada com fatores associados

\begin{tabular}{|c|c|c|c|c|}
\hline & RO (IC 95\%) & $p$ & RO* (IC 95\%) & $p$ \\
\hline \multicolumn{5}{|l|}{ Sexo } \\
\hline Masculino & 1.0 & & 1.0 & \\
\hline Feminino & $1.00(.72-1.40)$ & .975 & $1.07(.74-1.57)$ & .710 \\
\hline \multicolumn{5}{|l|}{ Faixa etária } \\
\hline $14-15$ & 1.0 & & 1.0 & \\
\hline $16-17$ & $1.03(.74-1.43)$ & .850 & $1.60(.77-3.29)$ & .200 \\
\hline \multicolumn{5}{|l|}{ Nível econômico } \\
\hline$A+B$ & $1.33(.88-2.01)$ & .173 & $2.04(.85-4.93)$ & .111 \\
\hline $\mathrm{C}$ & $.88(.60-1.28)$ & .496 & $1.15(.57-2.32)$ & .689 \\
\hline$D+E$ & 1.0 & & 1.0 & \\
\hline \multicolumn{5}{|l|}{ Área de domicílio } \\
\hline Urbana & $1.91(1.37-2.67)$ & $<.001$ & $1.91(1.36-2.69)$ & $<.001$ \\
\hline Rural & 1.0 & & 1.0 & \\
\hline \multicolumn{5}{|l|}{ Estado nutricional } \\
\hline Peso normal & 1.0 & & 1.0 & \\
\hline Baixo peso & $1.13(.71-1.80)$ & .592 & $1.10(.69-1.77)$ & .667 \\
\hline Excesso de peso & $.72(.38-1.39)$ & .332 & $.72(.37-1.39)$ & .333 \\
\hline
\end{tabular}

Nota: RO - Razão de odds; RO* - RO ajustada para todas as variáveis da tabela

Quanto aos indicadores da capacidade cardiorrespiratória, os escolares da área urbana apresentaram $91 \%$ mais chance de inadequação que àqueles da área rural. Esses achados são consistentes com os de outros estudos realizados com amostras de crianças e adolescentes brasileiros (Dumith, Azevedo Júnior, \& Rombaldi, 2008; Glaner, 2005). Outro estudo que verificou pior desempenho dos escolares da zona urbana em relação aos da zona rural, foi conduzido com escolares de 9 a 12 anos no México (Peña Reyes, Tan, \& Malina, 2003).

Os adolescentes urbanos apresentaram uma menor AFRS nas variáveis flexibilidade e aptidão cardiorrespiratória do que os respetivos pares rurais. Portanto, supõe-se que o estilo de vida, adotado por estes, contribui, sobremaneira, no desempenho dos testes. Apesar de não se ter sido verificado os possíveis fatores que influenciam nas performances dos testes, pode-se sugerir que se por um lado as atividades laborais, os hábitos e costumes cultivados na amostra rural interferem de forma positiva sobre a AFRS, por outro, o hábito de praticar atividades com pouco dispêndio energético, característica urbana, parece influenciar negativamente a AFRS da amostra citadina. Mesmo que a mecanização tenha diminuído o dispêndio energético na zona rural, ainda assim o gasto calórico pode ser considerado bem mais elevado em atividades laborais do que as realizadas em áreas urbanas (Montoye, Kemper, Saris, \& Washburn, 1996).

No indicador do componente da aptidão física relacionada à saúde força/resistência abdominal, os adolescentes da área rural apresentaram dez vezes mais chance de inadequação em relação aos seus pares da área urbana. Esta evidência causou certa surpresa, dado que se esperava que os adolescentes de zonas rurais fossem mais vigorosos. Não obstante, resultados semelhantes aos encontrados no presente estudo, foram observados em um estudo realizado com escolares do Rio Grande do Sul, onde os escolares da zona rural apresentaram piores desempenhos neste teste (Dumith et al., 2008). Em contrapartida, pesquisas realizadas com adolescentes rurais e urbanos do Norte gaúcho e Oeste catarinense 
revelaram piores desempenhos nos adolescentes da área urbana (Glaner, 2002, 2005).

Dentre as variáveis independentes estudadas (sexo, idade, nível econômico, área de domicílio e estado nutricional), a única que apresentou associação com os componentes de AFRS foi a área de domicílio, que explicou a maior parte da sua variação. Sexo, idade, nível econômico e estado nutricional, que podem ser tidos como variáveis contextuais, não se associaram com os componentes de AFRS. Isso sugere que o desempenho em testes que compõem a AFRS dos escolares do presente estudo pode estar mais relacionado às características propriamente ambientais.

Os resultados dos componentes da aptidão física relacionada à saúde não favorecem um único meio sociogeográfico, tendo a mesma situação sido registada em outros países. Em Portugal, os rapazes das zonas rurais demonstraram ser consistentemente superiores nas provas de força e velocidade, enquanto os seus pares do meio urbano se evidenciaram nas provas de agilidade e potência muscular (Rodrigues, Bezerra, \& Saraiva, 2005). Na Madeira, os rapazes do meio urbano e/ou semiurbano foram mais proficientes na flexibilidade, força e resistência muscular, enquanto os da área rural apresentaram melhores resultados na resistência aeróbia, e velocidade/agilidade. As moças do meio urbano e/ou semiurbano apresentaram melhores resultados na velocidade/agilidade, enquanto as do meio rural foram mais proficientes na força e resistência muscular (Ramos et al., 2008). Ainda em Portugal, Pereira e Soidán (2008) analisaram a aptidão física de crianças e adolescentes de duas regiões distintas para verificar o efeito da litoralidade/interioridade. Os autores não observaram um padrão de aptidão física, dado que os alunos da zona rural apresentam valores ligeiramente superiores nos componentes de força muscular, enquanto os da zona urbana foram superiores na flexibilidade, agilidade e resistência aeróbia. Em um estudo realizado na Grécia, também não foi observado predomínio da aptidão física em função do local de residência (Tsimeas, Tsiokanos, Koutedakis, Tsigilis, \& Kellis, 2005). Contudo, estudos epidemiológicos sugerem que altos níveis de aptidão física parecem retardar a mortalidade principalmente devido a taxas menores de doença cardiovascular e câncer (Blair et al., 1989).

As principais limitações do presente estudo estão relacionadas ao delineamento de corte transversal, que não permite identificar as relações de causalidade entre as variáveis e o uso de critérios referenciados à AFRS baseado em amostras de outros países. Outra limitação é a caracterização existente das áreas urbanas e rurais pelos municípios e IBGE. A população rural está sendo definida por exclusão: aquela que não habita as áreas urbanas. No entanto, muitos municípios brasileiros possuem ocupação urbana em áreas rurais (Caiado \& Santos, 2003). Além disso, destaca-se que o calor intenso da cidade pode ter prejudicado o desempenho nos testes motores.

\section{CONCLUSÕES}

Os resultados encontrados nesta pesquisa revelaram associação entre os componentes da AFRS e a área de domicílio. Esses achados permitem concluir que os adolescentes da área rural apresentaram melhores desempenhos na flexibilidade e aptidão cardiorrespiratória, enquanto na força/resistência muscular, uma melhor aptidão foi encontrada naqueles da área urbana. Por fim, adolescentes residentes tanto em áreas rurais quanto urbanas estão expostos aos fatores de risco relacionados à aptidão física relacionada à saúde.

Portanto, a escola tem um papel fundamental no desenvolvimento dos níveis de AFRS, através das aulas de Educação Física, visto que este espaço talvez se constitua como a única oportunidade que os adolescentes têm para participar de programas orientados de exercícios físicos.

A partir dos achados, sugere-se capacitação para os professores de Educação Física, diretores e supervisores, a fim de conscientizar sobre a importância de se trabalhar os componentes 
da AFRS para a promoção de saúde no âmbito escolar, bem como incentivar a prática da atividade física. Ainda, enfatiza-se a importância de realização de outras pesquisas, considerando aspetos como o estilo de vida, hábitos alimentares e prática regular de atividade física, para melhor compreender as questões que interferem na realidade encontrada.

\section{Agradecimentos:}

Nada a declarar.

\section{Conflito de Interesses:}

Nada a declarar.

\section{Financiamento:}

Nada a declarar.

\section{REFERÊNCIAS}

American Alliance for Health, Physical Education, Recreation and Dance - AAHPERD (1988). Physical best. Reston: Autor.

Associação Brasileira de Estudos Populacionais (2008). Critério de Classificação Econômica do Brasil. Disponível a partir de http://www.abep. org/codigosguias/ABEP_CCEB.pdf

Blair, S. N., Kohl, H. W., Paffenbarger, R. S., Clark, D. G., Cooper, K. H., \& Gibbons, L. W. (1998). Physical fitness and all-cause mortality: A prospective study of healthy men and women. The Journal of the American Medical Association, 262(17), 2395-2401. doi: 10.1001/jama.1989. 03430170057028

Caiado, A. S., \& Santos, S. M. (2003). Fim da dicotomia rural-urbano? Um olhar sobre os processos socioespaciais. São Paulo em Perspectiva, 17(3/4), 115-124. doi: 10.1590/S0102-883920 03000300012

Canadian Society for Exercise Physiology (2004). The Canadian physical activity, fitness and lifestyle appraisal: CSEP's guide to health active living ( $3^{\mathrm{a}}$ ed.). Ottawa: Autor.

Cole, T. J., Bellizzi, M. C., Flegal, K. M., \& Dietz, W. H. (2000). Establishing a standard definition for child overweight and obesity worldwide: International survey. British Medical Journal, 320 , 1-6. doi: 10.1136/bmj.320.7244.1240

Cole, T. J., Flegal, K. M., Nicholls, D., \& Jackson, A. A. (2007). Body mass index cut offs to define thinness in children and adolescents: Inter- national survey. British Medical Journal, 335, 194. doi: 10.1136/bmj.39238.399444.55

Dumith, S. C., Azevedo Júnior, M. R., \& Rombaldi, A. J. (2008). Aptidão física relacionada à saúde de alunos do ensino fundamental do município de Rio Grande, RS, Brasil. Revista Brasileira de Medicina do Esporte, 14(5), 454-459. doi: 10.159 0/S1517-86922008000500011

Glaner, M. F. (2002). Nível de atividade física e aptidão física relacionada à saúde em rapazes rurais e urbanos. Revista Paulista de Educação Física, 16(1), 76-85.

Glaner, M. F. (2005). Aptidão física relacionada à saúde de adolescentes rurais e urbanos em relação a critérios de referência. Revista Brasileira de Educação Física e Esporte, 19(1), 13-24.

Lopes, V. P., Maia, J. A., Silva, R. G., Seabra, A., \& Morais, F. P. (2004). Aptidão física associada à saúde da população escolar (6 a 10 anos de idade) do arquipélago dos Açores, Portugal. Revista Brasileira de Cineantropometria e Desempenho Humano, 6(1), 7-16.

Montoye, H. J., Kemper, H. C., \& Saris, W. H., \& Washburn, R. A. (1996). Measuring physical activity and energy expenditure. Champaign, IL: Human Kinetics.

Peña Reyes, M. E., Tan, S. K., \& Malina, R. M. (2003). Urban-rural contrasts in the physical fitness of school children in Oaxaca, Mexico. American Journal of Human Biology, 15(6), 800813. doi: 10.1002/ajhb.10218

Pereira, R. G., \& Soidán, J. L. (2008). Aptidão física, estudo de alunos do $3^{\circ}$ ciclo dos concelhos de Torre de Moncorvo e Vila Nova de Gaia. Motricidade, 4(1), 75-81.

Ramos, E. C., Freitas, D. L., Maia, J. A., Beunen, G. P., Claessens, A. L., Gouveia, E. R., ... Lefevre, J. (2008). Associação do envolvimento à actividade física e à aptidão em jovens madeirenses. Revista Portuguesa de Ciências do Desporto, 8(2), 229-240.

Rodrigues, L. P., Bezerra, P., \& Saraiva, L. (2005). Influência do meio (urbano e rural) no padrão de aptidão física de rapazes de Viana do Castelo, Portugal. Revista Portuguesa de Ciências do Desporto, 5(1), 77-84.

Ronque, E. R., Cyrino, E. S., Dorea, V. R., Serassuelo Junior, H., Galdi, E. H., \& Arruda, M. (2007). Diagnóstico da aptidão física em adolescentes de alto nível socioeconómico: Avaliação referenciada por critérios de saúde. Revista Brasileira de Medicina do Esporte, 13, 71-75. 
Serassuelo Junior, H., Rodrigues, A. R., Cyrino, E. S., Ronque, E. V., Oliveira, S. R., Simões, A. C. (2005). Aptidão física relacionada à saúde em escolares de baixo nível socioeconômico do município de Cambé/PR. Revista de Educação Física/UEM, 16(1), 5-11.

Silva, M. C., Paccini, M. K., \& Glaner, M. F. (2007). Aptidão física relacionada à saúde de adoles- centes oriundos de diferentes níveis econômicos. Revista de Educação Física/UEM, 18(2), 199-206.

Tsimeas, P. D., Tsiokanos, A. L., Koutedakis, Y., Tsigilis, N., \& Kellis, S. (2005). Does living in urban or rural settings affect aspects of physical fitness in children? An allometric approach. British Journal of Sports Medicine, 39(9), 671-674.

(cc) EY-NC Todo o conteúdo da revista Motricidade está licenciado sob a Creative Commons, exceto quando especificado em contrário e nos conteúdos retirados de outras fontes bibliográficas. 